\title{
Complicaciones postoperatorias en la herniorrafia ambulatoria con malla. Estudio comparativo de la tasa de infección del sitio operatorio con $y$ sin profilaxis antibiótica*
}

\author{
Drs. JORGE LEÓN S. ${ }^{1}$, ALBERTO ACEVEDO F. ${ }^{1}$, DAVID RIOSECO R. ${ }^{1}$, \\ JORGE VÁSQUEZ V. ${ }^{1}$, E.U. VERÓNICA DELLEPIANE ${ }^{1}$ \\ 1 Programa de Cirugía Ambulatoria de las Hernias, CRS Cordillera Oriente. \\ Universidad de Chile, Facultad de Medicina, Campus Oriente. \\ Santiago, Chile.
}

\begin{abstract}
Antibiotic prophylaxis in mesh inguinal herniorrhapy

The use of antibiotic prophylaxis has been questioned in the inguinal herniorrhaphy with mesh. The aim of this paper is to give account of the efficiency of antibiotic prophylaxis (AP) in the prevention of wound infection in the elective inguinal herniorrhaphy with mesh under local anaesthesia in an ambulatory basis. In the present observacional analytic study, 955 patients were operated upon with a mesh technique during a 10 year period, in the CRS Hernia Centre in Santiago. In the first group of 250 patients, 1 gr Cefazolin ${ }^{\circledR}$ was administered iv one hour before the operation, in the second group of 710 patients no antibiotics were used. Demographic variables, associated diseases, the length of surgery were comparable in both groups. The same team of surgeons work in both groups. Wound infection was defined as the presence of pus in the surgical wound associated with a positive bacterial culture. The rate of wound infections was $1.05 \%$ of the first and $0.35 \%$ of the second group. This difference was no significant $(\mathrm{p}<0.08)$. The isolated germ was a Staphylococcus aureus in all cases. The treatment was ambulatory in all cases. The rate of haematoma and funiculotesticular fluxion were low. We conclude that the rate of wound infection in mesh herniorrhaphies is low and that the antibiotic prophylaxis does not improve these results. Its routine use in these patients is not justified.
\end{abstract}

Key words: Inguinal herniorrhaphy, ambulatory surgery, local anaesthesia, antibiotic prophylaxis.

\section{Resumen}

Introducción: El uso de la profilaxis antibiótica en la cirugía herniaria con malla es controversial. El propósito del presente estudio es establecer la efectividad de la profilaxis antibiótica (PA) en la prevención de la infección del sitio operatorio (ISO) en la herniorrafia con malla efectuada en forma ambulatoria con anestesia local. Material y Método: El estudio observacional analítico se realizó dentro del Programa de Cirugía Ambulatoria del CRS Cordillera, en dos grupos consecutivos de pacientes, el primero que se sometió

*Recibido el 28 de Enero de 2010 y aceptado para publicación el 26 de Julio de 2010.

Correspondencia: Dr. Jorge León S.

Las Torres 5100, Peñalolén, Santiago, Chile.

jorgeleons@yahoo.com 
a PA mediante 1 gr de Cefazolina ${ }^{\circledR}$ intravenosa, inmediatamente antes del procedimiento quirúrgico, y el segundo en el que se prescindió de ella. Los controles postoperatorios se efectuaron al $7^{\circ}$ y $30^{\circ}$ día por un cirujano del grupo. Se diagnosticó ISO con la presencia de exudado purulento, con cultivo bacteriano positivo. Resultados: El estudio se efectuó en 955 pacientes operados entre 1998 y 2008. En el primer grupo, constituido por 245 pacientes se usó PA, en el segundo grupo de 710 pacientes se prescindió de ella. Las variables demográficas, antecedentes mórbidos y la duración del acto quirúrgico fueron comparables en ambos grupos. Se registraron 2 casos de infección en el primer grupo $(1,05 \%)$ y dos en el segundo $(0,35)$. Esta diferencia no fue significativa. El germen aislado fue en todos los casos el estafilococo dorado. Conclusiones: La tasa ISO en la herniorrafia inguinal electiva ambulatoria con malla es baja y no se modificó con el uso de PA. Su empleo indiscriminado no aparece justificado en estos pacientes.

Palabras clave: Cirugía ambulatoria, anestesia local, técnica de Lichtenstein, profilaxis antibiótica.

\section{Introducción}

La tasa de infección quirúrgica en cirugías limpias refleja la calidad de la técnica quirúrgica y las condiciones de higiene de un servicio de cirugía ${ }^{1-3}$. La infección depende de factores ambientales, de la afección quirúrgica y del huésped ${ }^{4}$, dentro de estos últimos se considera la diabetes mellitus y la obesi$\mathrm{dad}^{4}$. En cuanto a la contaminación, ésta proviene principalmente de la flora bacteriana de la piel del propio paciente, siendo el germen más frecuentemente aislado el Estafilococo dorado ${ }^{5}$.

En Chile, el Ministerio de Salud, ha establecido en el año 2005 una tasa nacional de $0,4 \%$ de infección del sitio operatorio (ISO) en la reparación de la hernia inguinal electiva de adultos ${ }^{5}$.

La profilaxis antibiótica es uno de los métodos más utilizados para prevenir esta complicación. Su utilidad se ha demostrado en las cirugías contaminadas ${ }^{4}$, no así en las cirugías limpias donde su uso es más controversial. Se ha recomendado su uso cuando existen factores de riesgo como la implantación de un material protésico, cuando la infección implica riesgo vital para el paciente, o frente a comorbilidades que impliquen un mayor riesgo de infección ${ }^{4}$.

La infección es poco probable en las herniorrafias inguinales con malla, dada la mínima cantidad de material protésico ("cuerpo extraño") utilizada. Esto es especialmente el caso cuando se utilizan mallas livianas y de malla amplia. Todo ello ha dado margen a que algunos centros prescindan sistemáticamente de la profilaxis antibiótica en las intervenciones de herniorrafia con prótesis ${ }^{6}$.

El objetivo del presente estudio, es dar a conocer los resultados de un estudio efectuado en la Unidad de Cirugía Mayor Ambulatoria del CRS Cordillera Oriente de la ciudad de Santiago, que compara la tasa ISO de pacientes sometidos a herniorrafia con malla con y sin profilaxis antibiótica.

\section{Material y Método}

Se trata de un estudio observacional analítico, efectuado en los pacientes sometidos a herniorrafia con malla en un período de 10 años (1998-2008) en el Programa de Cirugía Mayor ambulatoria del CRS Cordillera Oriente.

Se incluyeron en el presente estudio aquellos pacientes electivos portadores de una hernia inguinal primaria; con riesgo anestésico ASA I-II; que cumplía con los criterios para ser intervenidos en forma ambulatoria con anestesia local, y que se sometieron a una herniorrafia con malla. Se excluyeron pacientes con recidiva herniaria y con hernias femorales.

Los pacientes se agruparon según recibieran o no profilaxia antibiótica. En un primer grupo de 189 pacientes, intervenidos con anterioridad el 30 de junio de 2003, se utilizó profilaxis antibiótica con 1 gr de Cefazolina ${ }^{\circledR}$ iv 30 minutos antes de la intervención quirúrgica, y en un segundo grupo de 592 pacientes, intervenidos después del 1 de julio de 2003 se prescindió de ella.

Cada grupo se estratificó según la edad, sexo, ASA, tipo de hernia (directa o indirecta), presencia de Diabetes Mellitus, obesidad, técnica quirúrgica y el tiempo quirúrgico.

La anestesia local se efectuó por infiltración sucesiva de planos mediante Lidocaína ${ }^{\circledR}$ alcalinizada al $0,6 \%$, y con el agregado de Epinefrina ${ }^{\circledR}$. El volumen fue variable según la necesidad en cada caso.

Se estandarizó la técnica quirúrgica siguiendo los siguientes pasos según las recomendaciones de Lichtenstein y $\mathrm{Amid}^{7}$, con algunas modificaciones en el manejo del cremáster: Abierto el canal inguinal y manejado el cremáster se controló el cordón con una cinta de goma blanda y se expuso la pared posterior del canal inguinal. En las hernias directas se procedió a invaginar el saco mediante jareta de Vycryl ${ }^{\circledR}$ 2-0. En las hernias indirectas se disecó el saco hasta su base y tras desvirtualización se resecó o se redujo. Finalmente se cortó la malla para cubrir la pared 
posterior del canal inguinal y se fijó al tubérculo pubiano y al ligamento inguinal con puntos separados de Prolene ${ }^{\circledR}$ 2-0. La fijación a la vaina del recto se efectuó mediante puntos separados de Vycryl ${ }^{\circledR}$ 2-0. Las patas creadas por la sección proximal efectuada en la malla para dar paso al cordón se suturaron entre si con un punto de Prolene. Finalmente, en el segmento más proximal, se imbricó la pata medial sobre la lateral y se suturó con un punto de Prolene al ligamento inguinal. En los pacientes con grandes defectos del anillo inguinal profundo se colocó adicionalmente un tapón que se fijó a las estructuras vecinas con suturas de Prolene.

Todos los pacientes fueron intervenidos por un reducido grupo de cirujanos especializados en el tratamiento quirúrgico de las hernias de la pared abdominal

Desde el inicio del proyecto se utilizó un programa de vigilancia epidemiológica de las complicaciones postoperatorias y en especial de las ISO. El control se efectuó a las 24 horas, 7 y 30 días por un miembro del equipo quirúrgico, siguiendo un protocolo escrito cuyos resultados se vertieron en la base de datos del Programa de Cirugía de las Hernias del CRS. Se definió la infección de herida operatoria como la presencia de exudado purulento corroborada por un cirujano, y confirmada con un cultivo bacteriano.

Se consignó la fluxión funículo testicular en su forma severa, correspondiente a un engrosamiento del cordón mayor de $2 \mathrm{~cm}$. El diagnóstico de hematoma se realizó ante la presencia de un aumento de volumen circunscrito en la zona operatoria y/o el escroto acompañado de signos inflamatorios y cambios de coloración de la piel. En caso de duda se efectuó una punción evacuadora.

La probabilidad estadística de los resultados del estudio se estableció mediante el test de Chi cuadrado que es proporcionado en forma automática por el programa EpiStat del Epi Info 2002. Se consideró estadísticamente significativo un valor de $\mathrm{p}<0,05$.

\section{Resultados}

Se intervinieron 781 pacientes. El grupo con PA estuvo constituido por 189, el grupo sin PA por 592 pacientes. La proporción de varones fue similar en ambos grupos. No hubo una diferencia significativa en la edad, el género, el tipo de hernia inguinal y las comorbilidades de ambos grupos (Tabla 1).

La colocación de la malla se efectuó en todos los casos siguiendo las recomendaciones de Lichtenstein y Amid. Consideramos necesario instalar un tapón de Gilbert en el 13,9\% de los pacientes del grupo $1 \mathrm{y}$ en el $5,3 \%$ de los del grupo 2 . El tiempo promedio de cirugía fue de 40 minutos en ambos grupos (Tabla 2).
Una complicación se apreció en 58 pacientes, $7 \%$. Se apreció una infección de herida operatoria en dos casos del grupo control y en dos pacientes del grupo de los casos, con un $1,05 \%$ y un $0,34 \%$ respectivamente. Esta diferencia no fue significativa, con un $\mathrm{p}<0,08$. Las complicaciones se aprecian en la Tabla 3.

Tabla 1. Características demográficas y antecedentes mórbidos de ambos grupos de estudio

\begin{tabular}{lcc}
\hline Variable & $\begin{array}{c}\text { Grupo 1 } \\
\text { CPA (\%) }\end{array}$ & $\begin{array}{c}\text { Grupo 2 } \\
\text { SPA (\%) }\end{array}$ \\
\hline $\mathrm{N}^{\text {o de pacientes }}$ & 189 & 592 \\
Edad media (margen) & $54,5(22-87)$ & $51(15-87)$ \\
Varones & $171(90,5)$ & $517(87,3)$ \\
Mujeres & $18(9,5)$ & $75(12,7)$ \\
ASA I & $128(67,7)$ & $255(43,1)$ \\
ASA II & $61(32,3)$ & $337(56,9)$ \\
Inguinales directas & $110(58,2)$ & $342(57,8)$ \\
Indirectas & $79(41,8)$ & $250(42,3)$ \\
Diabetes Mellitus & $6(3,1)$ & $36(6,1)$ \\
Obesidad (IMC $>30)$ & $28(14,8)$ & $75(12,6)$ \\
\hline
\end{tabular}

CPA: Con profilaxis antibiótica. SPA: Sin profilaxis antibiótica.

Tabla 2. Características de técnica quirúrgica en ambos grupos de estudio

\begin{tabular}{lcc}
\hline Variable & $\begin{array}{c}\text { Grupo 1 } \\
\text { CPA (\%) }\end{array}$ & $\begin{array}{c}\text { Grupo 2 } \\
\text { SPA (\%) }\end{array}$ \\
\hline $\mathrm{N}^{\text {o de pacientes }}$ & 189 & 592 \\
Tiempo quirúrgico & $40 \mathrm{~min}$ & $40 \mathrm{~min}$ \\
Lichtenstein (Licht) & $179(94,7)$ & $510(86,1)$ \\
Licht + Tapón & $10(5,3)$ & $82(13,9)$ \\
Anestesia local & $189(100)$ & $592(100)$ \\
\hline
\end{tabular}

Tabla 3. Complicaciones postoperatorias en ambos grupos del estudio

\begin{tabular}{lcc}
\hline Variable & $\begin{array}{c}\text { Grupo 1 } \\
\text { CPA (\%) }\end{array}$ & $\begin{array}{c}\text { Grupo 2 } \\
\text { SPA (\%) }\end{array}$ \\
\hline $\mathrm{N}^{\text {o de pacientes }}$ & 189 & 592 \\
Infección herida operatoria & $2(1,05)$ & $2(0,3)$ \\
Hematoma & $8(4,2)$ & $20(3,3)$ \\
Fluxión testicular & $4(2,1)$ & $22(3,7)$ \\
\hline
\end{tabular}


Todas las infecciones se diagnosticaron en el control del séptimo día. No hubo infecciones en los obesos ni en los diabéticos. En todos los casos el microorganismo cultivado fue un Estafilococo dorado.

Los pacientes fueron tratados en forma ambulatoria con drenaje de la herida y curaciones periódicas. Se utilizó Cloxacilina ${ }^{\circledR}$ a dosis habituales por 7 días. En ninguno de los casos fue necesario retirar la malla.

La presencia de hematomas y de fluxión funículotesticular en la muestra se aprecia en la tabla 3. Sólo 2 hematomas requirieron de un tratamiento quirúrgico con drenaje precoz de la colección y cierre primario. Ambos permanecieron hospitalizados 1 día y evolucionaron favorablemente. La fluxión funículo testicular se manifestó, habitualmente, en el control semanal, desarrollándose hasta alcanzar el tamaño definitivo en las 2 ó 3 semanas siguientes. Estos pacientes se controlaron semanalmente y su tratamiento fue conservador.

\section{Discusión}

En Chile, la tasa acumulada de infección en los pacientes adultos sometidos a herniorrafia entre 2003 y 2005 fue de $0,65 \%$. Este estudio no especifica si hubo reparaciones con malla en la muestra estudiada. Sobre esta base, el MINSAL, establece un indicador de ISO en la cirugía de hernia inguinal en adultos de 1,1 , vigente desde el 1 de enero de $2007^{9}$.

Un meta-análisis de infección de la herniorrafia inguinal utilizando material protésico mostró tasas entre 0,1 y el $9 \%{ }^{8}$.

Altemaier ${ }^{10}$, publicó en 1987 su índice, señalando como valores máximos aceptables de infección en heridas limpias una tasa inferior al $5 \%$; esta cifra ha experimentado un progresivo descenso aceptándose hoy en día tasas inferiores al $2 \%{ }^{3}$. Estas cifras serían razón más que suficiente, para el uso de profilaxis antibiótica en las herniorrafias cuando se utiliza material protésico ${ }^{11}$, aunque estudios efectuados en herniorrafias con y sin malla comprobaron tasas de infección similares para ambos grupos ${ }^{12,13}$.

En un meta análisis realizado por Sánchez et al, en 12 estudios randomizados, con un total de 6.705 pacientes (4.128 con profilaxis antibiótica y 2.577 sin profilaxis) se estableció que la tasa de infección fue similar en ambos grupos. Concluyeron que la administración de antibióticos profilácticos no estaba justificada en reparación herniaria electiva ${ }^{14}$.

En otro meta análisis realizado por el grupo de Aufenacker ${ }^{15}$, se concluye que la profilaxis antibiótica no previene ni reduce significativamente la ocurrencia de infecciones en la cirugía de la hernia inguinal.
El mismo autor realizó un estudio multicéntrico, doble ciego, randomizado controlado entre noviembre de 1997 y mayo de 2003, donde analizó 1008 pacientes operados por técnica de Lichtenstein, randomizados: un grupo con una dosis única de 1,5 gr IV de Cefotaxima y otro con placebo. Hubo 8 infecciones $(1,6 \%)$ en el grupo con profilaxis y 9 $(1,8 \%)$ en el grupo placebo $(p=0,82)$. Concluyendo que el porcentaje de infecciones en la reparación primaria por técnica de Lichtenstein es baja y que no existen diferencias entre el grupo que se realizó profilaxis y el placebo. Estos hallazgos sugieren no ser necesario el uso antibiótico profiláctico en este tipo de intervenciones ${ }^{16}$.

Pérez et al, realizaron un estudio prospectivo, randomizado, doble ciego de la tasa de infección en 360 pacientes con herniorrafia de Lichtenstein (180 con profilaxis antibiótica y 180 con placebo). Los resultados obtenidos en este estudio tampoco avalan el uso de profilaxis antibiótica en hernioplastías con mallas $^{17}$

Más recientemente, Tzovaras, realizó un estudio similar al de Pérez, comparando dos grupos de 193 pacientes cada uno con características similares. Se comprobaron 5 infecciones en el grupo con profilaxis y 9 en el placebo con un $p<0,4$, no significativo. Establece que la profilaxis antibiótica no ofrece beneficio alguno en la cirugía electiva de la hernia inguinal con malla ${ }^{18}$.

Estos antecedentes bibliográficos fueron los que nos movieron a diseñar un estudio prospectivo destinado a establecer la efectividad de la profilaxis antibiótica en la prevención de la ISO. Los dos grupos de pacientes de este estudio son considerablemente asimétricos y ello se debe a que no siendo un estudio randomizado no vimos la necesidad de que los grupos fueran de tamaño similar.

Otra circunstancia que nos motivó a efectuar este estudio fue la comprobación en el programa de vigilancia epidemiológica de las ISO, que realizamos desde el inicio del Programa de Cirugía Ambulatoria de las Hernias, de una tasa de infección alrededor del $0,5 \%$ a pesar de la profilaxis antibiótica.

Nuestra serie no difiere de series extranjeras en cuanto a las variables demográficas de los grupos, las condiciones mórbidas asociadas, ASA y el tipo de hernia ${ }^{11-19}$. Tampoco existen diferencias respecto al tiempo operatorio promedio y las complicaciones. Nuestros resultados avalan la falta de efectividad de la profilaxis antibiótica en las herniorrafias con malla. En nuestro estudio el grupo sin profilaxis antibiótica tuvo una tasa de ISO $0,3 \%$, menor que la del grupo con profilaxis que tuvo una tasa de $1,05 \%$. (Tabla 3).

En un paciente la infección derivó en una fístula rebelde al tratamiento conservador que obligó a una 
intervención para retirar la malla infectada. Ello se efectuó igualmente en forma ambulatoria, con anestesia local.

La mayor proporción de hernias directas observada en este estudio se debe a que hemos colocado mallas con mayor frecuencia en este tipo de hernias. Analizando el total de las hernias inguinales intervenidas en el programa de cirugía ambulatoria se comprueba que las hernias directas y las indirectas están representadas con una frecuencia similar.

Llama la atención el bajo porcentaje de complicaciones apreciado en los pacientes de nuestra serie (Tabla 3). A la baja tasa de infección que alcanzó una tasa de $0,4 \%$ para ambos grupos en conjunto, deben agregarse tasas de hematoma de $3,6 \%$ y de fluxión funículo-testicular de $3,1 \%$ para la totalidad la cohorte. Ello es la consecuencia de diversas medidas dentro de las cuales mencionamos la ambulatoriedad, el uso de anestesia local, una técnica disectiva cuidadosa y la especialización en cirugía herniaria del grupo quirúrgico.

El mayor riesgo de hacer infecciones de los pacientes diabéticos y de los obesos establecido en el memorando del MINSAL en Chile ${ }^{4}$, no se confirmó en nuestra muestra en la que ninguno de los pacientes infectados era diabético u obeso. Estos resultados se contravienen, también, con los resultados dados a conocer por Aufenacker et $\mathrm{al}^{16}$, que lo llevan a recomendar la exclusión de estos pacientes de los programas quirúrgicos con alta precoz.

Concluimos que la herniorrafia con malla, ambulatoria, con anestesia local presenta bajas tasas de complicaciones postoperatorias. El uso de profilaxis antibiótica en la hernioplastia inguinal primaria con malla no determinó una baja en la tasa de ISO y su uso rutinario no está justificado.

\section{Referencias}

1. Cruse PJE. The epidemiology of wound infection. Surg Clin North AM 1980;60:27-40.

2. Cruse PJE. Incidence of wound infection on the surgical services: Surg Clin NA. 1975;55:1269-75.

3. Bannura G, Guerra, JF, Salvado J. Infección de la herida operatoria en hernioplastía inguinal primaria. Rev Chil Cir. 2006;58:330-5.

4. Prevención y control de infección de herida operatoria o sitio quirúrgico. Gobierno de Chile Ministerio de Salud, 2004.
5. Informe vigilancia epidemiológica de las infecciones intrahospitalarias. Chile 2005. MINSAL.

6. Aranaz JM, Romero M, Compaña AF, Candela A, Marco J, Aranaz J, y cols. Cirugía Española 2003;74:97-103.

7. Amid PK, Shulman AG, Lichtenstein IL. Critical scrutiny of the open tension-free hernioplasty. Am J Surg. 1993;165:369-71.

8. Granell J. Prótesis de pared abdominal e infección quirúrgica. Cir Esp. 1998;63:333-5.

9. Indicadores de Infecciones Intrahospitalarias. MINSAL. 2006.

10. Altemeier W: Manual de control de la infección en pacientes quirúrgicos. $2^{\mathrm{a}}$ ed. Am Coll Surg Ed Interam Española, 1987.

11. Yerdel MA, Akin EB, Dolalan S. Effect of SingleDose Prophylactic Ampicillin and Sulbactam on Wound Infection After Tensión-Free Inguinal Hernia Repair With Polypropylene Mesh. Ann Surg. 2001;233:26-33.

12. Gilbert AI, Felton LL. Infeccion in inguinal hernia repair considering biomaterials and antibiotics. Surg Gynecol Obstet. 1993;177:126-30.

13. Thill RH, Hopkins WM. The use of Mersilene mesh in adult inguinal and femoral hernia repairs: a comparison with classic techniques. Am Surg. 1994;60:553-6.

14. Sánchez-Manuel FJ, Lozano-García J, Seco-Gil JL. Antibiotic prophylaxis for hernia repair. Cochrane Database of Systematic Reviews 2007.

15. Aufenacker TJ, Koelemay MJ, Gouma DJ, Simons MP. Systematic review and meta-analysis of the effectiveness of antibiotic prophylaxis in prevention of wound infection after mesh repair of abdominal wall hernia. $\mathrm{Br}$ J Surg. 2006;93:5-10.

16. Aufenacker TJ, van Geldere D, van Mesdag T, Bossers AN, Dekker B, Scheijde E, et al. The role of antibiotic prophylaxis in prevention of wound infection after Lichtenstein open mesh repair of primary inguinal hernia: a multicenter double-blind randomized controlled trial. Ann Surg. 2004;240:955-60; discussion 960-1.

17. Pérez AR, Roxas MF, Hilvano SS. A randomized, double-blind, placebo-controlled trial to determine effectiveness of antibiotic prophylaxis for tension-free mesh herniorrhaphy. J Am Coll Surg. 2005;200:393-7.

18. Tzovaras G, Delikoukos S, Christodoulides G, Spyridakis M, Mantzos F, Tepetes K, et al. The role of antibiotic prophylaxis in elective tension-free mesh inguinal hernia repair: results of a single-centre prospective randomised trial. Int J Clin Pract. 2007;61:236-9.

19. Bringman S, Ramel S, Heikkinen TJ. Tension-Free Inguinal Hernia Repair: TEP versus Mesh-Plug, versus Lichtenstein. Ann Surg 2003;237:142-7. 